\title{
RADIOGRAPHIC EVALUATION OF TWO TREATMENT MODALITIES OF RESORBED MANDIBULAR RIDGE
}

\author{
Noha H. Nawar* and Shaimaa Lotfy**
}

\begin{abstract}
Objectives: The treatment plan is responsible for the design, number and position of the implants so the purpose of this study was to radiographically evaluate the success of two treatment options to overcome anatomical obstacles in resorbed mandible involving installing distal short implants versus All on 4 concept.
\end{abstract}

Materials and Methods: Fourteen completely edentulous patients were selected according to certain criteria, and they were randomly allocated into two equal groups, Group I: patients had guided surgical installation of four implants following "All on 4 "concept and Group II: patients had guided surgical installation of two straight interforaminal implants $(3.5 \times 11 \mathrm{~mm})$ and two distal short implants $(4 \times 4 \mathrm{~mm})$. The implants in the two groups were monitored using cone beam computed tomography on loading, 6 month and one year later.

Results: In this study, at the end of follow up period, there was statistically significant difference in the marginal bone height loss between the two group. The least bone loss was reported around the implants in group II.

Conclusion: Within the limitation of the number of patients and the follow-up periods enrolled in this study, it was concluded that widely spread of implants is much better than limiting the spread of implants even for the same number of implants. Recommendation: The authors recommend further investigation on All on 4 concept with longer follow up periods

Key Words: Dental Implant, All on 4, short implant, AP spread, cantilever length

\section{INTRODUCTION}

Complete maxillary and mandibular dentures have been the traditional solution for edentulous patients for more than a century. Completely edentulous patients are usually able to wear an upper denture without problems, but many struggles to perform normal functions with the complete lower denture because of its inherit diminished retention and stability. (1) The implants represent a significantly better solution for tooth loss replacement, as they are anchored directly into the bone and provide complete stability in contrast to the traditional tooth-replacement alternatives. ${ }^{(2)}$

* Associate Professor of Removable Prosthodontics, Faculty of Dentistry Ain Shams University.

** Lecturer of Removable Prosthodontics, Faculty of Dentistry Ain Shams University. 
Installation of one to four implants are indicated to improve the stability and retention of a conventional complete denture. Two designs may be applied either unsplinted (single attachments) or splinted designs (bar systems). The design of the overdenture prosthesis must be carefully planned according to the requirements to ensure adequate stability and optimal form, contour, esthetics, and the patient's best comfort. ${ }^{(3)}$ Some patients reject the idea of two implant supported overdenture as from their point of view that this option still lies under the category of removable prosthesis.

In completely edentulous patients, there are some anatomical obstacles that cause implant supported prosthesis treatment to be almost impossible without complex techniques such as nerve transposition and grafting in the posterior maxilla and mandible. ${ }^{(4)} \mathrm{A}$ solution for such situations is the All-on-4 concept, this method advocates tilting distal implants in edentulous arches which permits the placement of longer implants, improved prosthetic support with shorter cantilever arm, improved inter implant distance and anchorage in the bone. The "All-on-4 "treatment concept was developed by Paulo Malo(5) with straight and angled multi-unit abutments, to provide edentulous patients with an immediately loaded full arch restoration with only four implants. Long-time denture wearers are often challenged because of severe bone loss. Having

Tilting of posterior implants make it possible to achieve good bone anchorage without interfering with mental foramina in the mandible and are an alternative to sinus floor augmentation in severely resorbed maxillae. ${ }^{(6)}$ All-on-4 concept can support non-removable teeth using just 4 strategicallyplaced implants. By positioning the implanted portions at unique angles, the strategic anatomical landmarks were protected. Also, the biting and chewing forces are distributed evenly among fewer implants and the minimal amount of bone becomes no longer a problem. ${ }^{(7)}$
Short dental implants were defined as implants with an endosseous component of $\leq 8 \mathrm{~mm}$. ${ }^{(8)}$ These implants were predominantly introduced and clinically used for circumventing more extensive primary bone augmentation procedures such as lateral sinus augmentation. ${ }^{(9)}$ However, the survival rates were found to be lower than those of standardlength implants in identical clinical situations. Further, the development of new implant surfaces resulted in an increasing number of publications using short dental implants for many indications, thereby expanding the treatment options for fully and partially edentulous patients. From a clinical point of view and from a patient's perspective, short dental implants offer many clinical advantages: less skill necessary to perform the surgical intervention, less morbidity by avoiding more extensive bone augmentation procedures, easier removal in case of failure, and predominantly an increased number of sites available for implant therapy. In contrast, clinicians might be worried about disadvantages including a high crown-to-implant ratio and a relatively high rate of biological and technical complications associated with potential overload. ${ }^{(10-12)}$

The main objective of this study was to radiographically evaluate the success of two treatment options to overcome anatomical obstacles in resorbed mandible involving installing distal short implants versus All on 4 concept and to weight All on 4 as a treatment modality in comparison to other traditional modalities.

\section{MATERIALS AND METHODS}

\section{Participants:}

Fourteen edentulous patients ranged between 50 and 60 years were selected from outpatient clinic of Prosthodontic Department, Faculty of Dentistry, Ain Shams University. Patients were included in this study according to the following criteria: healthy mucosa, complete edentulism for at least 1-year, 
Angle class I ridge relationship, sufficient bone height and width (at least $6 \mathrm{~mm}$ width posteriorly and $5.5 \mathrm{~mm}$ anteriorly while having at least $12 \mathrm{~mm}$ bone height). The following conditions were excluded: diabetes, smoking habit, osteoporosis, immune deficiency, radiotherapy to the head and neck region and anticoagulant therapy. After the patients were informed about the line of treatment and the need for frequent recalls throughout the total period of the research, they all signed a written consent.

\section{Patients grouping:}

The patients were then randomly assigned using random number generator and checker (www. psychicscience.org/random.aspx) into two equal groups:

Group I: Patients rehabilitated with mandibular implant retained overdenture using four implants installed following All on 4 concept with the distal implants tilted 30 degrees

Group II: Patients rehabilitated with mandibular implant retained overdenture using two interforaminal implants $(3.5 \times 11 \mathrm{~mm})$ and two short distal implants $(4 \mathrm{x} 4 \mathrm{~mm})$

All patients received new complete conventional dentures constructed with bilateral balanced occlusal scheme and were allowed at least 2 months to wear the prostheses prior to implant surgery. Double scanning protocol was followed to construct completely limiting CAD-CAM surgical guide following the treatment plan for each group. Gutta Percha markers were inserted on several random positions and in different axial planes on the polished surface of the denture, then a CBCT was made to the patient while wearing the denture. Then another scan was made for the denture only.

The OnDemand3D computer software was used to select the implant sites according to the optimum site anatomically and functionally, also the location of the three anchor pins were selected to be on three widely separated positions to be used for fixation of the guide. Once the planning was done, the OnDemand3D software created the guide from the scanned denture design and was saved as "STL" file (Standard triangular language) to be sent to the Envisiontec digital dental 3D printing machine (Envisiontec, Dearborn, MI, USA) to fabricate a clear surgical guide with a metal sleeves inserted to guide the implant drilling. (Fig 1)

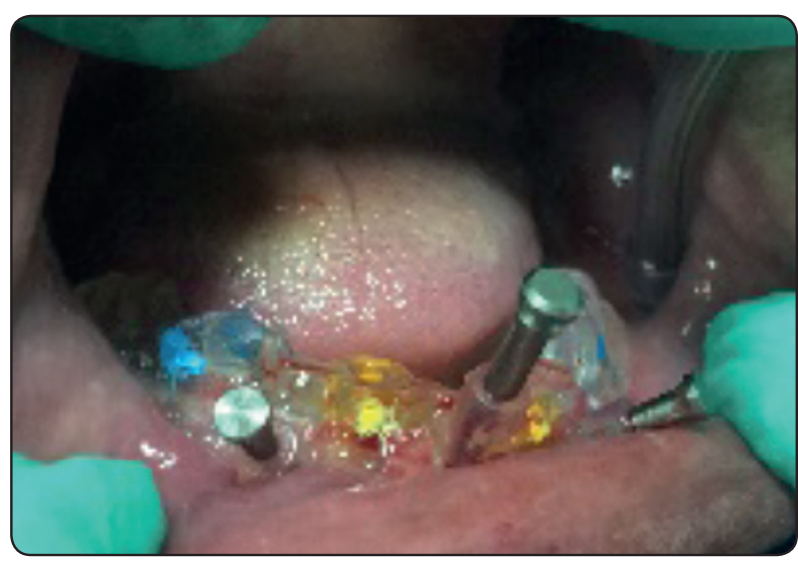

Fig. (1) Surgical guide in the patient mouth

\section{Surgical and Prosthetic procedure:}

\section{For group I:}

The surgical procedures were performed under local anesthesia according to the planning done before. Four implants (Texas MC, Leader, Italy) were installed in interforminal area with the distal implants tilted 30 degrees. Sequential drilling was done after tissue punching and fixation of the surgical guide using fixative pins. Countersinking was performed when needed to create space for the head of the tilted implants. The implant head neck was aimed to be positioned at bone level. The patients were recalled after one week to install the abutments provided that the implant stability reading was more than 50. ${ }^{(13)}$ Straight, and $30^{\circ}$ angulated abutments with different collar heights were placed onto the implants. These are used to achieve the 
correct access allowing relative parallelism and so that the rigid prosthesis can be seated passively. Then the provisional complete denture was used to pick up of the attachment intraorally using self-cure acrylic resin. (Fig 2)

\section{FOR GROUP II:}

The surgical procedure was done as group I but for installing two implants at site of laterals $(3.5 \times 11$ $\mathrm{mm})$ and two distal short implants at the site of first molars $(4 \times 4 \mathrm{~mm})$ and then the same procedure were carried out for group II patients. The female metal housings were fixed on the ball attachment and the denture was positioned back in place to assure complete seating and complete relief around the balls and the housings. Any additional adjustments needed were done. A special celluloid ring was fixed around the neck of each implant and edges of the female housing to prevent possible leak of self-cure acrylic resin and its hardening around the neck of the ball attachment or edges of the housing (Fig 3).

All patients were included in a plaque control protocol, and this was reinforced at subsequent reviews (every 3 months). At the post-operative visits, occlusion was checked as well as the need for any prosthetic adjustments.

\section{Radiographic evaluation}

Marginal bone height change around the implants was evaluated using the linear measurement system supplied by the cone beam computed tomography.

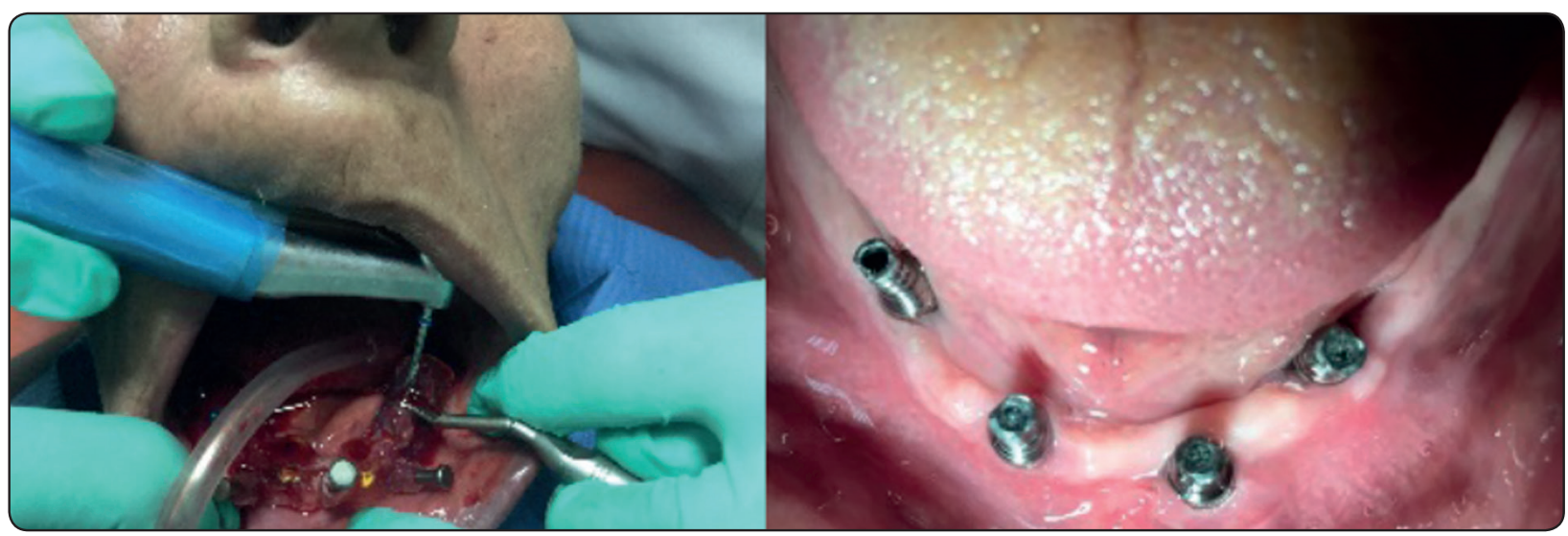

Fig. (2) installing implants for group I intreorally

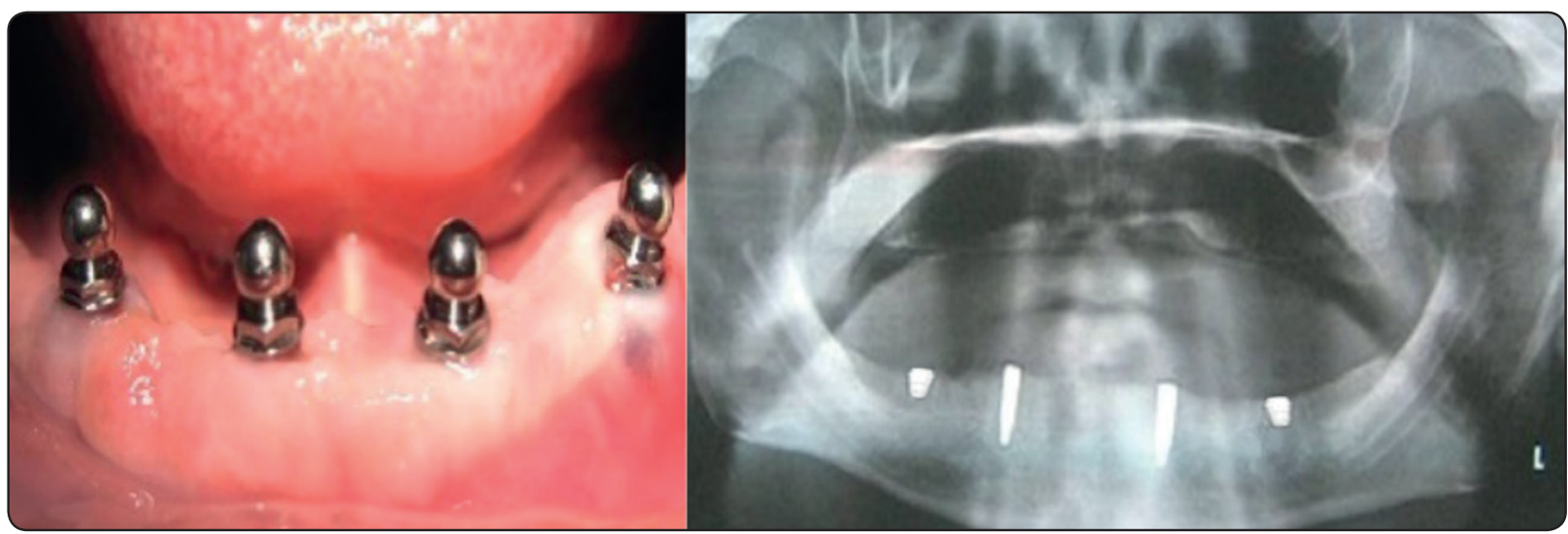

Fig. (3) Ball attachments secured into implants \&radiographic view of group II 
Marginal bone height changes around each implant were monitored. A ruler in the software was used to measure the bone height from the apex of the implant to crestal bone in contact with the implant.

The measurements were carried out at the end of each follow up appointment (at insertion, 6, and 12 months post insertion). The marginal bone loss at different intervals was obtained by calculating the difference in bone height at that interval from the base line measurement. (Fig 4)

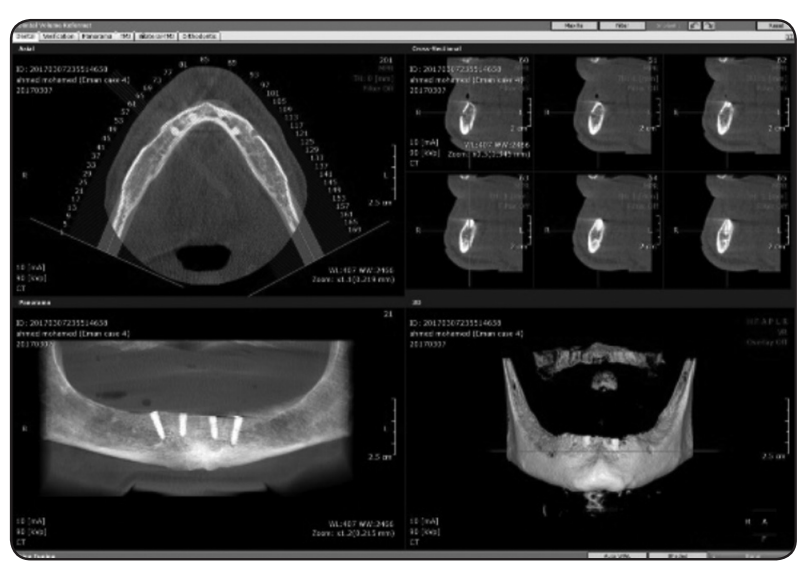

Fig. (4) CBCT measurement of marginal bone heights

\section{RESULTS}

Numerical data were explored for normality by checking the data distribution, calculating the mean and median values, evaluating histograms and normality curves and using Kolmogorov-Smirnov and Shapiro-Wilk tests. Data were presented by mean, standard deviation (SD). Independent $t$ test was used for comparison between groups. Paired $t$ test was used for comparison between follow up period. The significance level was set at $\mathrm{P} \leq 0.05$. Statistical analysis was performed with IBM $^{\circledR}$ SPSS $^{\circledR}$ Statistics Version 20 for Windows.

As confirmed in table 1 throughout the whole follow up period there was statistically significant difference between the two groups with the least mean difference within group II.

In this study, statistical analysis revealed that the bone height changes by time within each group were statistically significant from time of loading to six months and from six months to one year with least mean difference bone height change from six to one year.

TABLE (1): The mean differences, standard deviation (SD) values and comparison between amounts of bone loss around the two group at direct intervals.

\begin{tabular}{|l|c|c|c|c|c|}
\hline \multirow{2}{*}{\multicolumn{1}{c|}{ Intervals }} & \multicolumn{2}{c|}{ Group I } & \multicolumn{2}{c|}{ Group II } & \multirow{2}{*}{ P value } \\
\cline { 2 - 5 } & Mean & SD & Mean & SD & \\
\hline Time of loading -six months & .3732 & .0578 & .2761 & .0673 & $0.013^{*}$ \\
\hline Six months-one year & .2686 & .0403 & .1432 & .0594 & $<0.001^{*}$ \\
\hline Time of loading -one year & .6418 & .0839 & .4193 & .1039 & $0.001^{*}$ \\
\hline
\end{tabular}

TABLE (2): The mean differences, standard deviation (SD) values and results of paired t-test for the changes by time in mean bone height within each group

\begin{tabular}{|c|c|c|c|c|c|}
\hline \multirow{2}{*}{} & \multicolumn{2}{|c|}{ Time of loading -six months } & \multicolumn{2}{|c|}{ Six months-one year } & \multirow{2}{*}{ P value } \\
\cline { 2 - 5 } & Mean & SD & Mean & .0403 & 0.002 \\
\hline Group I & .3732 & .0578 & .2686 & .0594 & 0.003 \\
\hline Group II & .2761 & .0673 & .1432 & .20 \\
\hline
\end{tabular}




\section{DISCUSSION}

Prosthodontists faced several problems in rehabilitation of completely edentulous mandibular ridge because of limited supporting area and the inherited problems of complete denture, so this study was carried out to discuss two different treatment options and their validity.

Planning implant position, size, number, direction, and placement depended on the presurgical diagnostic radiographing, which often, was limited to two-dimensional radiographs, and on the guiding acrylic stents that usually prepared over duplicated casts of diagnostic wax-up. However, limitations of two-dimensional imaging and inaccuracies in the stent fabrication often lead to defective implant placement, which results in complications and implant failure, especially in severely resorbed mandible. Recent advanced technology trying to offer new solutions in treatment planning to overcome these limitations, which have computerized the implant-dentistry and introducing guided surgical implant installment. ${ }^{(14,15)}$

A wide variety of different sizes and shapes of implants have been introduced to go with current surgical concepts and improve patient treatment. Continuous research proved that changes in shape, length, and width of the implants could affect success rates. ${ }^{(16,17)}$

Research in implant dentistry proved that length ensure implant success so longer implants guarantee higher success rates, due to increasing implantbone interface. ${ }^{(16-18)}$ However, short implants are preferred for the prosthetic solution of the extremely resorbed alveolar bone areas. ${ }^{(19)}$ It was proved that width of implant is far more important than its length in controlling stresses specially in cortical bone. ${ }^{(20)}$ this could support the result of this study in addition to the antero-posterior spread (AP spread) which was on the side of group II rather than group I.

In case of multiple implants supported prosthesis, the wide spread of implants and stiffness of the prosthesis will reduce bending of the implant. (21) The more distal position of the posterior implant and the resulting shorter cantilever may have a role in reduction of stress values in the implant. ${ }^{(22)}$

Some authors used provisional prostheses and others recommended splinted frameworks when implants are immediately loaded. This is for both axial and tilted implants. In- vitro studies on tilted implants showed an increase in the stress transferred to the bone. The implants may also have subjected to bending during function, which may lead to increased marginal bone loss. This is also agreed with the results of this study that showed more marginal bone loss in group II than group I. ${ }^{(23,24)}$

In general data and long follow ups of All on 4 concept in the literature are still scarce however when used in the mandible, tilting of posterior implants makes it possible to achieve good bone anchorage without interfering with mental foramina in severely resorbed ridge ${ }^{(6)}$ but its success depends on guarding against long cantilever and an anteroposterior spread that minimizes the distal cantilevers and establishes well distributed four-point stability was probably contributory to both implant and prosthetic success. ${ }^{(25)}$

\section{CONCLUSION}

Within the limitation of the number of patients and the follow-up period enrolled in this study, it was concluded that widely spread of implants is much better than limiting the spread of implants even for the same number of implants. Recommendation: The authors recommend further investigation on All on 4 concept with longer follow up periods.

\section{REFERENCES}

1- Feine JS, Carlsson GE, Awad MA. The McGill Consensus Statement on Overdentures. Montreal, Quebec, Canada. Inter. J. Prosthodont. 2002; 15:413-4.

2- Carlsson GE. Facts and Falacies:An evidence base for complete dentures. Dent Update. 2006; 33:134-142.

3- Zitzmann NU, Marinello CP ,A review of clinical and technical considerations for fixed and removable implant 
prostheses in the edentulous mandible. Int J Prosthodont. $2002 ; 15(1): 65-72$.

4- Ellingsen JE, Johansson CB, Wennerberg A, Holmén A. Improved retention and bone-to implant contact with fluoride-modified titanium implants. Int J Oral Maxillofac Implants 2004; 19:659-66.

5- Paulo Malo, Bo Rangert, Miguel Nobre. All-on- $4{ }^{\circledR}$ immediate function concept for completely edentulous maxillae. A clinical report on the medium and long term 5 years outcome. J Oral Implant. 2011, 37 (4):87-95.

6- Taruna M, Chittaranjan B, Sudheer N, Tella S, Abusaad M. Prosthodontic Perspective to All-On-4® Concept for Dental Implants. J Clin Diagn Res. 2014;8(10): 16-19.

7- Grandi T, Guazz6-i P, Samarani R, Grandi G. Immediate Loading of four All-on-4® Post-extractive implants supporting mandibular cross arch fixed prosthesis - 18 months follow up from a multi centre prospective cohort. Eur J Oral Implant. 2012;5(3):277-85.

8. Renouard F, Nisand D. Impact of implant length and diameter on survival rates. Clin Oral Implants Res 2006;17 (2):35-51.

9. Renouard F, Nisand D. Short implants in the severely resorbed maxilla: a 2-year retrospective clinical study. Clin Implant Dent Relat Res 2005;7 (1):S104-10.

10. Gotfredsen K, Berglundh T, Lindhe J. Bone reactions adjacent to titanium implants subjected to static load of different duration. A study in the dog (III). Clin Oral Implants Res 2001;12:552-8.

11. Gotfredsen K, Berglundh T, Lindhe J. Bone reactions adjacent to titanium implants with different surface characteristics subjected to static load. A study in the dog (II). Clin Oral Implants Res 2001;12:196-201.

12. Gotfredsen K, Berglundh T, Lindhe J. Bone reactions adjacent to titanium implants subjected to static load. A study in the dog (I). Clin Oral Implants Res 2001;12:1-8.

13- Krafft T, Graef F, Karl, M. Osstell Resonance Frequency Measurement Values as a Prognostic Factor in Implant Dentistry. J. Oral Implantol.2015;41, 133-137 .

14- Winter AA, Pollack AS, Frommer HH, Koenig L. Cone beam volumetric tomography vs. medical CT scanners. N Y State Dent J 2005; 71:28-33.
15- Kobayashi K, Shimoda S, Nakagawa Y, Yamamoto A. Accuracy in measurement of distance using limited cone-beam computerized tomography. Int $\mathrm{J}$ Oral Maxillofac Implants 2004;19:228-31.

16- Searson LJ. History and development of dental implants. In: Narim L, Wilson HF, eds. Implantology in general dental practice. London, Chicago: Quintessence Publishing Co; 2005:19-41.

17- Mijiritsky E, Mazor Z, Lorean A, Levin L. Implant diameter and length influence on survival: interim results during the first 2 years of function of implants by a single manufacturer. Implant Dent 2013;22:394-8.

18- Lee JH, Frias V, Lee KW, Wright RF. Effect of implant size and shape on implant success rates: a literature review. J Prosthet Dent 2005;94:377-81.

19- Mandhane SS, More AP. A review: evaluation of design parameters of dental implant abutment. Inter J Emerging Sci Eng 2014;2:64-7.

20- Himmlová L, Dostálová T, Kácovský A, Konvicková $\mathrm{S}$. Influence of implant length and diameter on stress distribution: a finite element analysis. J Prosthet Dent 2004; 91:20-5.

21- Dallenback K, et al. Biomechanics of in-line vs offset implants supporting a partial prosthesis. J Dent Res. 1996;75:183.

22- Bellini Chiara M, et al. Comparison of tilted versus non-tilted Implant -supported prosthetic designs for the restoration of the edentulous mandible. A biomechanical study. Int J oral Maxillofacial implants. 2009;24:511-12.

23- Watanabe F, Hata Y, Komatsu S, Ramos TC, Fukuda H. Finite element analysis of the influence of implant inclination, loading position, and load direction on stress distrubtion. Odontology 2003;91:31-36.

24- Khatami AH, Smith CR. All-on-Four immediate function concept and clinical report of treatment of an edentulous mandible with a fixed complete denture and milled titanium framework. J Prothodont 2008; 17: 47-51.

25- Guilherme Carvalho Silva, et al. Stress patterns on implants in prostheses supported by four or six implants. A three dimensional finite element analysis. Int J Oral Maxillofac Implants. 2010;25:239-46.[PubMed] 